\title{
Nonparametric Regression with Subfractional Brownian Motion via Malliavin Calculus
}

\author{
Yuquan Cang, Junfeng Liu, and Yan Zhang \\ School of Mathematics and Statistics, Nanjing Audit University, 86 West Yushan Road, Pukou, Nanjing 211815, China \\ Correspondence should be addressed to Junfeng Liu; jordanjunfeng@163.com
}

Received 27 November 2013; Accepted 13 December 2013; Published 6 February 2014

Academic Editor: Litan Yan

Copyright (C) 2014 Yuquan Cang et al. This is an open access article distributed under the Creative Commons Attribution License, which permits unrestricted use, distribution, and reproduction in any medium, provided the original work is properly cited.

We study the asymptotic behavior of the sequence $S_{n}=\sum_{i=0}^{n-1} K\left(n^{\alpha} S_{i}^{H_{1}}\right)\left(S_{i+1}^{H_{2}}-S_{i}^{H_{2}}\right)$, as $n$ tends to infinity, where $S^{H_{1}}$ and $S^{H_{2}}$ are two independent subfractional Brownian motions with indices $H_{1}$ and $H_{2}$, respectively. $K$ is a kernel function and the bandwidth parameter $\alpha$ satisfies some hypotheses in terms of $H_{1}$ and $H_{2}$. Its limiting distribution is a mixed normal law involving the local time of the sub-fractional Brownian motion $S^{H_{1}}$. We mainly use the techniques of Malliavin calculus with respect to sub-fractional Brownian motion.

\section{Introduction}

The asymptotic theory of nonlinear regression plays a central role in econometrics, underlying models as diverse as simultaneous equations systems and discrete choice. Two examples in econometrics are GMM estimation and nonlinear cointegration. GMM limit theory was originally developed for ergodic and strictly stationary time series (Hansen [1]) for which all measurable functions are stationary and ergodic, so that applications of strong laws and central limit theory are straightforward. Although some attempts have been made to extend the theory to models with deterministically trending data (e.g., Andrewa and McDermott [2] and Wooldridge [3]), traditional CLT approaches have still been used and no significant progress has been made. Nonlinear cointegrating models also seem important in a range of applications (e.g., Granger [4]) and models with nonlinear attractor sets have been popular in economics for many years.

The motivation of this paper comes from the econometric theory. Consider a nonlinear structural model of cointegration

$$
y_{t}=f\left(x_{t}\right)+u_{t}, \quad t=1,2, \ldots, n,
$$

where $u_{t}$ is a stationary equilibrium error process, $x_{t}$ is a jointly dependent nonstationary regressor, and $f$ is an unknown function to be estimated with the observed data $\left\{y_{t}, x_{t}\right\}_{t=1}^{n}$. Let $K(x)$ be a nonnegative real function and set
$K_{h}(s)=(1 / h) K(s / h)$, where the bandwidth parameter $h \equiv$ $h_{n} \rightarrow 0$. The conventional kernel estimate of $f(x)$ in model (1) is given by

$$
\widehat{f}(x)=\frac{\sum_{t=1}^{n} y_{t} K_{h}\left(x_{t}-x\right)}{\sum_{t=1}^{n} K_{h}\left(x_{t}-x\right)} .
$$

The limit behavior of $\widehat{f}(x)$ has recently been investigated in Karlsen et al. [5] in the situation where $x_{t}$ is a recurrent Markov chain. The main theorem in Karlsen et al. ([5], Theorem 3.1) relies on the asymptotic theory developed in Karlsen et al. [5] involving the conditions on the invariant measure associated with a recurrent Markov chain. These conditions are not always easy to check in practice and do not include some cases of econometric interest such as fractional processes. Wang and Phillips [6] provided an alternative approach to nonparametric cointegration in developing the asymptotics. In particular, instead of the recurrent Markov chain in Karlsen et al. [5]), they worked with partial sum representations of the type $x_{t}=\sum_{j=1}^{t} \xi_{j}$, where $\xi_{j}$ is a Gaussian process or a general linear process, and obtained the limit behavior for kernel functions of this process. This specification corresponds to the conventional formulation of unit root and cointegration models, and the limit theory has links to traditional nonparametric asymptotics for stationary models even though rates of convergence are different. This approach also allows them to work with cases where the 
regressor $x_{t}$ is a non-stationary long memory time series. An important assumption in the main part of the above references is the fact that the error process $u_{t}$ is a martingale difference sequence and there is no contemporaneous correlation between $x_{t}$ and $u_{t}$.

The estimation error in the kernel estimator (2) has the usual decomposition

$$
\begin{aligned}
\hat{f}(x)-f(x)= & \frac{\sum_{t=1}^{n} u_{t} K_{h}\left(x_{t}-x\right)}{\sum_{t=1}^{n} K_{h}\left(x_{t}-x\right)} \\
& +\frac{\sum_{t=1}^{n}\left[f\left(x_{t}\right)-f(x)\right] K_{h}\left(x_{t}-x\right)}{\sum_{t=1}^{n} K_{h}\left(x_{t}-x\right)} .
\end{aligned}
$$

The second term of (3) affects bias, and, at least when this is of smaller order, it is the first term that determines the asymptotic distribution. The asymptotic behavior of the estimator $\hat{f}(x)$ is usually related to the behavior of the sequence

$$
M_{n}=\sum_{t=1}^{n} u_{t} K_{h}\left(x_{t}-x\right) .
$$

The limit in distribution as $n \rightarrow \infty$ of the sequence $M_{n}$ has been widely studied in the literature in various situations. Inspired by Bourguin and Tudor [7], we will consider the following situation: we assume that the regressor $x_{t}=S_{t}^{H_{1}}$ is a subfractional Brownian motion with Hurst index $H_{1} \in(0,1)$ and the error is $u_{t}=S_{t+1}^{H_{2}}-S_{t}^{H_{2}}$, where $S_{t}^{H_{2}}$ is a sub-fractional Brownian motion with $\mathrm{H}_{2} \in(0,1)$ which is independent of $S_{t}^{H_{1}}$. In this case, our error process is no semimartingale property. We will set $h_{n}=n^{-\alpha}$ with $\alpha>0$. A supplementary assumption on $K$ will be imposed later in terms of the Hurst parameters $H_{1}$ and $H_{2}$. The sequence $M_{n}$ can be now written as

$$
S_{n}(x)=\sum_{t=1}^{n} K\left(n^{-\alpha}\left(S_{t}^{H_{1}}-x\right)\right)\left(S_{t+1}^{H_{2}}-S_{t}^{H_{2}}\right) .
$$

Our purpose is to give an approach based on stochastic calculus for this asymptotic theory. Recently, the stochastic integration with respect to the sub-fractional Brownian motion has been widely studied (see Yan et al. [8, 9] and references therein). Various types of stochastic integrals, based on Malliavin calculus, has been introduced and change of variables formulas have been derived. We will use all these different techniques in our work. The general idea is as follows. Suppose that $x=0$. We will first observe that the asymptotic behavior of the sequence $S_{n}$ will be given by the sum

$$
\begin{array}{rl}
N_{n}=\sum_{j, k=0}^{n} & K\left(n^{\alpha} S_{j}^{H_{1}}\right) K\left(n^{\alpha} S_{k}^{H_{1}}\right) \\
& \times E\left(\left(S_{j+1}^{H_{2}}-S_{j}^{H_{2}}\right)\left(S_{k+1}^{H_{2}}-S_{k}^{H_{2}}\right)\right) .
\end{array}
$$

This is easy to understand since the conditional distribution of $S_{n}$ given $S^{H_{1}}$ is given by $\sqrt{N_{n}} Z$, where $Z$ is a standard normal random variable. The double sum $N_{n}$ can be decomposed into two parts: a "diagonal" part and a "nondiagonal" part. We will restrict ourselves to the situation where the diagonal part is dominant (in a sense that will be defined later) with respect to the non-diagonal part. This will imply a certain assumption on the bandwidth parameter $K$ in terms of $H_{1}$ and $H_{2}$. We will therefore need to study the asymptotic behavior of

$$
\langle S\rangle_{n}=\sum_{j=1}^{n} K^{2}\left(n^{\alpha} S_{j}^{H_{1}}\right) .
$$

In the case $H_{2}=1 / 2$ this is actually the bracket of $S_{n}$ which is a martingale; this motivates our choice of notation. We will assume that the kernel $K$ is the standard Gaussian kernel

$$
K(x)=\frac{1}{\sqrt{2 \pi}} e^{-x^{2} / 2} .
$$

This choice is motivated by the fact that $K^{2}\left(n^{\alpha} S_{j}^{H_{1}}\right)$ can be decomposed into an orthogonal sum of multiple Wiener-Itô integrals and the Malliavin calculus can be used to treat the convergence of (7). Its limit in distribution will be after normalization of the local time of the sub-fractional Brownian motion denoted by $c \mathscr{L}^{H_{1}}(1,0)$, where $c$ is positive constant. Consequently, we will find that the (renormalized) sequence $S_{n}$ converges in law to a mixed normal random variable $c W_{\mathscr{L}^{H_{1}}(1,0)}$, where $W$ is a Brownian motion independent of $S^{H_{1}}$ and $c$ is a positive constant.

We also mention that, although the error process $S^{H_{2}}$ does not appear in the limit of (5), it governs the behavior of this sequence. Indeed, the parameter $\mathrm{H}_{2}$ is involved in the renormalization of (5) and the stochastic calculus with respect to $S^{H_{2}}$ is crucial in the proof of our main results.

We have organized our paper as follows. Section 2 contains the notations, definitions, and results from the stochastic calculus that will be needed throughout our paper. In Section 3 we will find the renormalization order of the sequence (5), while Section 4 contains the result of the convergence of the "bracket" (7). In Section 5 we will prove the limit theorem in distribution for $S_{n}(0)$.

\section{Preliminaries and Notations}

In this section we describe the elements from stochastic calculus that we will need in the paper. Let $S^{H}=\left\{S_{t}^{H}, t \in\right.$ $[0, T]\}$ be a standard subfractional Brownian motion (subfBm for short) with parameter $H \in(0,1)$. It is well known that this process is a centered Gaussian process with the following covariance function:

$$
\begin{aligned}
C_{H}(s, t) & \equiv E\left[S_{t}^{H} S_{s}^{H}\right] \\
& =s^{2 H}+t^{2 H}-\frac{1}{2}\left[(s+t)^{2 H}+|t-s|^{2 H}\right],
\end{aligned}
$$

for all $s, t \geq 0$. For $H=1 / 2, S^{H}$ coincides with the standard Brownian motion $B . S^{H}$ is neither a semimartingale nor a Markov process unless $H=1 / 2$, so many of the powerful techniques from stochastic analysis are not available when dealing with $S^{H}$. The subfBm has properties analogous to 
those of fBm (self-similarity, long-range dependence, and Hölder paths) and satisfies the following estimates:

$$
\begin{array}{r}
\left(2-2^{2 H-1}\right)(t-s)^{2 H} \leq E\left[\left(S_{t}^{H}-S_{s}^{H}\right)^{2}\right] \leq(t-s)^{2 H}, \\
\text { if } H>\frac{1}{2}, \\
(t-s)^{2 H} \leq E\left[\left(S_{t}^{H}-S_{s}^{H}\right)^{2}\right] \leq\left(2-2^{2 H-1}\right)(t-s)^{2 H}, \\
\text { if } H<\frac{1}{2} .
\end{array}
$$

But its increments are not stationary; more works for subfractional Brownian motion can be found in Bojdecki et al. [10-12], Liu et al. [13, 14], Tudor [15], and Yan et al. [8, 9].

Consider $\mathscr{H}_{H}$ a real separable Hilbert space and $\left\{S^{H}(\varphi), \varphi \in \mathscr{H}_{H}\right\}$ an isonormal Gaussian process defined on a complete probability space $(\Omega, \mathscr{B}, P)$, that is, centered Gaussian family of random variables such that $E\left(S^{H}(\varphi) S^{H}(\psi)\right)=$ $\langle\varphi, \psi\rangle_{\mathscr{H}_{H}}$. In this case for sub-fractional Brownian motion, the space $\mathscr{H}_{H}$ is the canonical Hilbert space of the subfractional Brownian motion which is defined as the closure of the linear space generated by the indicator functions $\left\{1_{[0, t]}, t \in[0, T]\right\}$ with respect to the scalar product

$$
\left\langle 1_{[0, t]}, 1_{[0, s]}\right\rangle_{\mathscr{H}_{H}}=C_{H}(s, t), \quad s, t \in[0, T] .
$$

Denote by $I_{n}\left(f_{n}\right)$ the multiple Itô stochastic integral of a symmetric kernel $f_{n} \in L^{2}\left([0, T]^{n}\right)$, with respect to $S^{H}$. This $I_{n}$ is actually an isometry between the Hilbert $\mathscr{H}_{H}^{\circ n}$ (symmetric tensor product) equipped with the norm $(1 / \sqrt{n !})\|\cdot\|_{\mathscr{H}_{H}^{\otimes n}}$ and the $n$th Wiener chaos which is defined as the closed linear span of the random variables $H_{n}\left(S^{H}(\varphi)\right)$, where $\varphi \epsilon$ $\mathscr{H}_{H},\|\varphi\|_{\mathscr{H}_{H}}=1$, and $H_{n}$ is the Hermite polynomial of degree $n \geq 1$ defined for $n \geq 1$ by

$$
H_{n}(x)=\frac{(-1)^{n}}{n !} \exp \left(\frac{x^{2}}{2}\right) \frac{d^{n}}{d x^{n}}\left(\exp \left(-\frac{x^{2}}{2}\right)\right), \quad x \in \mathbb{R},
$$

and $H_{0}(x)=1$. The isometry of multiple integrals can be written as follows for positive integers $m, n$,

$$
E\left(I_{m}(f) I_{n}(g)\right)= \begin{cases}n !\langle f, g\rangle_{\mathscr{H}_{H}^{\otimes n},} & \text { if } m=n, \\ 0, & \text { if } m \neq n .\end{cases}
$$

It also holds that

$$
I_{n}(f)=I_{n}(\tilde{f})
$$

where $\tilde{f}$ denotes the symmetrization of $f$ defined by

$$
\tilde{f}\left(t_{1}, \ldots, t_{n}\right)=\frac{1}{n !} \sum_{\sigma \in \mathcal{S}_{n}} f\left(t_{\sigma(1)}, \ldots, t_{\sigma(n)}\right) .
$$

Recall that the Wiener chaos expansion of a square integrable Brownian random variable $F$ is given by

$$
F=\sum_{n=0}^{\infty} I_{n}\left(f_{n}\right)
$$

where $f_{n} \in \mathscr{H}_{H}^{\otimes n}$ are symmetric functions and $I_{0}\left(f_{0}\right)=E[F]$.
Let $\mathbf{L}$ be the Ornstein-Uhlenbeck operator

$$
\mathbf{L F}=-\sum_{n=0}^{\infty} n I_{n}\left(f_{n}\right)
$$

where $F$ is given by (16). If $p \in(1, \infty)$ and $\alpha \in \mathbb{R}$, we define the Sobolev-Watanabe spaces $\mathbb{D}^{\alpha, p}$ as the closure of the set of polynomial random variables with respect to the norm

$$
\|F\|_{\alpha, p}=\|(\mathbf{I d}-\mathbf{L})\|_{L^{p}(\Omega)}
$$

where Id stands for the identity mapping.

Let us denote by $\mathcal{S}$ the set of smooth functionals of the form

$$
F=f\left(S^{H}\left(\varphi_{1}\right), S^{H}\left(\varphi_{2}\right), \ldots, S^{H}\left(\varphi_{n}\right)\right),
$$

where $f \in C_{b}^{\infty}\left(\mathbb{R}^{n}\right)$ and $\varphi_{i} \in \mathscr{H}$. The Malliavin derivative $D$ of a functional $F$ as above is given by

$$
D F=\sum_{j=1}^{n} \frac{\partial f}{\partial x_{j}}\left(S^{H}\left(\varphi_{1}\right), S^{H}\left(\varphi_{2}\right), \ldots, S^{H}\left(\varphi_{n}\right)\right) \varphi_{j} .
$$

The derivative operator $D$ is then a closable operator from $L^{2}(\Omega)$ into $L^{2}\left(\Omega ; \mathscr{H}_{H}\right)$. We denote by $\mathbb{D}^{1,2}$ the closure of $\mathcal{S}$ with respect to the norm

$$
\|F\|_{1,2}:=\sqrt{E|F|^{2}+E\|D F\|_{\mathscr{H}_{H}}^{2}} .
$$

The divergence integral $\delta^{H}$ is the adjoint operator of $D$. That is, we say that a random variable $u$ in $L^{2}\left(\Omega ; \mathscr{H}_{H}\right)$ belongs to the domain of the divergence operator $\delta^{H}$, denoted by $\operatorname{Dom}\left(\delta^{H}\right)$, if

$$
E\left|\langle D F, u\rangle_{\mathscr{H}_{H}}\right| \leq c\|F\|_{L^{2}(\Omega)},
$$

for every $F \in \mathcal{S}$. In this case $\delta^{H}(u)$ is defined by the duality relationship

$$
E\left[F \delta^{H}(u)\right]=E\langle D F, u\rangle_{\mathscr{H}_{H}}
$$

for any $u \in \mathbb{D}^{1,2}$. We have $\mathbb{D}^{1,2} \subset \operatorname{Dom}\left(\delta^{H}\right)$. We will use the notation

$$
\delta^{H}(u)=\int_{0}^{T} u_{s} d S_{s}^{H}
$$

to express the Skorohod integral of a process $u$ with respect to $S^{H}$.

Let $u$ be a stochastic process having the chaotic decomposition $u_{t}=\sum_{n \geq 0} I_{n}\left(f_{n}(\cdot, t)\right)$, where $f_{n}(\cdot, t) \in \mathscr{H}_{H}^{\otimes n}$ for every $s$. One can prove that $u \in \operatorname{Dom}\left(\delta^{H}\right)$ if and only if $\widetilde{f}_{n} \in \mathscr{H}_{H}^{\otimes(n+1)}$ for every $n \geq 0$, and $\sum_{n \geq 0} I_{n+1}\left(\widetilde{f}_{n}\right)$ converges in $L^{2}(\Omega)$. In this case,

$$
\begin{gathered}
\delta^{H}(u)=\sum_{n \geq 0} I_{n+1}\left(\tilde{f}_{n}\right), \\
E\left(\delta^{H}(u)\right)^{2}=\sum_{n \geq 1}(n+1) !\left\|\tilde{f}_{n}\right\|_{\mathscr{H}_{H}^{\otimes(n+1)}}^{2} .
\end{gathered}
$$


We denote by $D$ the derivative operator, defined on multiple integrals as

$$
D_{t} I_{n}\left(f_{n}\right)=n I_{n-1}\left(f_{n}(\cdot, t)\right)
$$

This operator is continuous from $\mathbb{D}^{\alpha, p}$ into $\mathbb{D}^{\alpha-1, p}\left(L^{2}([0, T])\right)$. It is known that a random variable $F$ belongs to $\mathbb{D}^{\alpha, 2}$, if and only if its chaotic decomposition $\sum_{n=0}^{\infty} I_{n}\left(f_{n}\right)$ satisfies

$$
\sum_{n=0}^{\infty}(1+n)^{\alpha}\left\|I_{n}\left(f_{n}\right)\right\|_{2}^{2}<\infty
$$

Set $\mathbb{D}^{\infty, 2}=\bigcap_{\alpha \in \mathbb{R}} \mathbb{D}^{\alpha, 2}$. The Stroock formula that gives the Wiener chaos decomposition of a functional $F \in \mathbb{D}^{\infty, 2}$ is

$$
F=\sum_{n=0}^{\infty} \frac{1}{n !} I_{n}\left(\mathbb{E}\left(D^{n} F\right)\right) .
$$

For a complete survey of these materials we refer the reader to the book by Nualart [16].

\section{Renormalization of the Sequence $S_{n}$}

We will assume throughout the paper that $x=0$ in (5); then

$$
S_{n}:=S_{n}(0)=\sum_{j=0}^{n-1} K\left(n^{\alpha} S_{j}^{H_{1}}\right)\left(S_{j+1}^{H_{2}}-S_{j}^{H_{2}}\right)
$$

We compute in this part the $L^{2}$-norm of $S_{n}$ in order to renormalize it. We have

$$
\begin{aligned}
E\left(S_{n}^{2}\right)= & E\left(\sum_{j, k=0}^{n-1} K\left(n^{\alpha} S_{j}^{H_{1}}\right) K\left(n^{\alpha} S_{k}^{H_{1}}\right)\left(S_{j+1}^{H_{2}}-S_{j}^{H_{2}}\right)\right. \\
& \left.\times\left(S_{k+1}^{H_{2}}-S_{k}^{H_{2}}\right)\right) \\
= & E\left(\sum_{j=0}^{n-1} K^{2}\left(n^{\alpha} S_{j}^{H_{1}}\right)\left(S_{k+1}^{H_{2}}-S_{k}^{H_{2}}\right)^{2}\right) \\
& +E\left(\sum_{j \neq k}^{n-1} K\left(n^{\alpha} S_{j}^{H_{1}}\right) K\left(n^{\alpha} S_{k}^{H_{1}}\right)\left(S_{j+1}^{H_{2}}-S_{j}^{H_{2}}\right)\right. \\
:= & A+B .
\end{aligned}
$$

The summand $A$ will be called the "diagonal" term while the summand $B$ will be called the "non-diagonal" term. We will analyze each of them separately.

Let $C_{H}(s, t)$ be the covariance function of the $\operatorname{subfBm} S^{H}$, and set

$$
Q_{H}(z) \begin{cases}\frac{C_{H}(1, z)}{z^{H}}, & \text { if } z \in(0,1] \\ 0, & \text { if } z=0 .\end{cases}
$$

In the following we will denote that $\beta_{H}=2-2^{2 H-1}$ and $\beta_{H_{i}}=2-2^{2 H_{i}-1}, i=1,2$. The following lemma is due to (68) in Yan and Shen [8].

Lemma 1. For all $s, t \in[0, T], s<t$, and $0<H<1$, then one has

$$
\begin{aligned}
\left(1-2^{H-1}\right)^{2}(t-s)^{2 H} s^{2 H} & \leq \beta_{H}^{2} s^{2 H} t^{2 H}-\mu^{2} \\
& \leq 2\left(2+2^{H}\right)(t-s)^{2 H} s^{2 H},
\end{aligned}
$$

where $\mu=E\left(S_{s}^{H} S_{t}^{H}\right)$.

Lemma 2. Let $0 \leq s \leq t \leq T$; one has

$$
0 \leq C_{H}(t, s) \leq \frac{1}{2} \beta_{H}\left[t^{2 H}+s^{2 H}-|t-s|^{2 H}\right],
$$

for $1 / 2<H<1$.

Proof. Make the change of variable $s=t x$ with $0 \leq x \leq 1$. One can easily show that the function

$$
\begin{aligned}
f(x):= & 1+x^{2 H}-\frac{1}{2}\left[(1+x)^{2 H}+(1-x)^{2 H}\right] \\
& -\frac{1}{2}\left(2-2^{2 H-1}\right)\left[1+x^{2 H}-(1-x)^{2 H}\right]
\end{aligned}
$$

is nonpositive by convexity. This completes the proof.

The following lemma, which will be needed in the sequel, studies the properties of the function $Q_{H}(\cdot)$ and the behavior of $\int_{0}^{1} Q_{H}(z)^{n} z^{-H} d z$ when $n$ goes to infinity.

Lemma 3. There exists a constant $C_{H}$, independent of $n$, such that

$$
\int_{0}^{1} \frac{1+x^{2 H}-(1-x)^{2 H}}{2^{n} x^{H(n+1)}} d x \leq C_{H} n^{-1 / 2 H}
$$

for all integers $n \geq 1$, where $K_{H}$ is positive constant depending only on $\mathrm{H}$.

Combining Lemmas 2 and 3, we can easily check the following estimate:

$$
\int_{0}^{1} Q_{H}(z)^{n} z^{-H} d z \leq C_{H} n^{-1 / 2 H}
$$

Concerning the term $A$ in (30) we have the following.

Proposition 4. As n tends to infinity,

$$
\frac{\left[\beta_{H_{2}} \wedge 1\right]}{2 \pi \sqrt{2 \beta_{H_{1}}}\left(1-H_{1}\right)} \leq \lim _{n \rightarrow \infty} n^{\alpha+H_{1}-1} A \leq \frac{\left[\beta_{H_{2}} \vee 1\right]}{2 \pi \sqrt{2 \beta_{H_{1}}}\left(1-H_{1}\right)}
$$

Proof. Due to the independence of $S^{H_{1}}$ and $S^{H_{2}}$, we have that

$$
A=\left(\sum_{i=0}^{n-1} E\left(K^{2}\left(n^{\alpha} S_{j}^{H_{1}}\right)\right) E\left(S_{j+1}^{H_{2}}-S_{j}^{H_{2}}\right)^{2}\right) .
$$


We denote that $A_{1}=\sum_{j=0}^{n-1} E\left(K^{2}\left(n^{\alpha} S_{j}^{H_{1}}\right)\right)$ for convenience. Then

$$
\begin{aligned}
A_{1} & =\sum_{j=0}^{n-1} E\left(K^{2}\left(n^{\alpha} \sqrt{\beta_{H_{1}}} j^{H} \xi\right)\right) \\
& =\sum_{j=0}^{n-1} E\left(\frac{1}{2 \pi} e^{-n^{2 \alpha} \beta_{H_{1}} j^{2 H_{1}} \xi^{2}}\right),
\end{aligned}
$$

where $\xi$ is a standard normal random variable. Recall that, if $\xi$ is a standard normal random variable and if $1+2 c>0$,

$$
E\left(e^{-c \xi^{2}}\right)=\frac{1}{\sqrt{1+2 c}}
$$

Consequently

$$
A_{1}=\sum_{j=0}^{n-1} \frac{1}{2 \pi} \frac{1}{\sqrt{1+2 n^{2 \alpha} \beta_{H_{1}} j^{2 H_{1}}}} .
$$

Then

$$
\begin{aligned}
A= & \left(\sum_{i=0}^{n-1} E\left(K^{2}\left(n^{\alpha} S_{j}^{H_{1}}\right)\right) E\left(S_{j+1}^{H_{2}}-S_{j}^{H_{2}}\right)^{2}\right) \\
= & \sum_{j=0}^{n-1} \frac{(2 j)^{2 H_{2}}}{2 \pi} \frac{1}{\sqrt{1+2 n^{2 \alpha} \beta_{H_{1}} j^{2 H_{1}}}} \\
& \times\left[\left(1+\frac{1}{2 j}\right)^{2 H_{2}}-\frac{1}{2}\left(1+\frac{1}{j}\right)^{2 H_{2}}-\frac{1}{2}+\left(\frac{1}{2 j}\right)^{2 H_{2}}\right] \\
= & \sum_{j=0}^{n-1} \frac{(2 j)^{2 H_{2}}}{2 \pi} \frac{1}{\sqrt{1+2 n^{2 \alpha} \beta_{H_{1}} j^{2 H_{1}}}} \\
& \times\left[-\frac{H_{2}\left(2 H_{2}-1\right)}{4} \frac{1}{j^{2}}+\frac{1}{(2 j)^{2 H_{2}}}+o\left(j^{-2}\right)\right] .
\end{aligned}
$$

As $n$ tends to infinity, the term $A$ behaves as such

$$
\begin{aligned}
\sum_{j=0}^{n-1} \frac{1}{2 \pi} & \frac{1}{\sqrt{1+2 n^{2 \alpha} \beta_{H_{1}} j^{2 H_{1}}}} \\
& \sim \frac{n^{-\alpha}}{2 \pi \sqrt{2 \beta_{H_{1}}}} \sum_{j=0}^{n-1} j^{-H_{1}} \\
& \sim \frac{n^{1-\alpha-H_{1}}}{2 \pi \sqrt{2 \beta_{H_{1}}}} \frac{1}{n} \sum_{i=0}^{n-1}\left(\frac{i}{n}\right)^{-H_{1}} \\
& \sim \frac{n^{1-\alpha-H_{1}}}{2 \pi \sqrt{2 \beta_{H_{1}}}} \int_{0}^{1} x^{-H_{1}} d x \\
\sim & \frac{n^{1-\alpha-H_{1}}}{2 \pi \sqrt{2 \beta_{H_{1}}}\left(1-H_{1}\right)},
\end{aligned}
$$

where the sign " $\sim$ " means that the left-hand side and the right-hand side have the same limit as $n$ tends to infinity. We will use this sign throughout this paper. Hence

$$
\lim _{n \rightarrow \infty} n^{\alpha+H_{1}-1} A \text { exists. }
$$

Since

$$
\beta_{\mathrm{H}_{2}} \wedge 1 \leq E\left(S_{j+1}^{H_{2}}-S_{j}^{H_{2}}\right)^{2} \leq \beta_{H_{2}} \vee 1,
$$

so

$$
\begin{aligned}
{\left[\beta_{H_{2}}\right.} & \wedge 1] E\left(K^{2}\left(n^{\alpha} S_{j}^{H_{1}}\right)\right) \\
& \leq A \leq \sum_{j=0}^{n-1} E\left(K^{2}\left(n^{\alpha} S_{j}^{H_{1}}\right)\right)\left[\beta_{H_{2}} \vee 1\right] .
\end{aligned}
$$

Thus, according to expression (47), the proof is complete.

We now compute the term $B$.

Proposition 5. Suppose

$$
\alpha-4 H_{2}+H_{1}+4>0 .
$$

Then, as $n$ tends to infinity,

$$
\lim _{n \rightarrow \infty} n^{\alpha+H_{1}-1} B=0 .
$$

Proof. Using again the independence of $S^{H_{1}}$ and $S^{H_{2}}$,

$$
\begin{aligned}
B= & \sum_{j \neq k}^{n-1} E\left(K\left(n^{\alpha} S_{j}^{H_{1}}\right) K\left(n^{\alpha} S_{k}^{H_{1}}\right)\right) \\
& \times E\left(S_{j+1}^{H_{2}}-S_{j}^{H_{2}}\right)\left(S_{k+1}^{H_{2}}-S_{k}^{H_{2}}\right) \\
= & \sum_{j \neq k}^{n-1} E\left(K\left(n^{\alpha} S_{j}^{H_{1}}\right) K\left(n^{\alpha} S_{k}^{H_{1}}\right)\right) f_{H_{2}}(j, k),
\end{aligned}
$$

where

$$
\begin{aligned}
f_{\mathrm{H}_{2}}(j, k)=\frac{1}{2}[ & 2(j+k+1)^{2 \mathrm{H}_{2}}+(k+1-j)^{2 \mathrm{H}_{2}} \\
+ & (k-1-j)^{2 \mathrm{H}_{2}}-(k+j+2)^{2 \mathrm{H}_{2}} \\
& \left.-2(k-j)^{2 \mathrm{H}_{2}}-(k+j)^{2 \mathrm{H}_{2}}\right] .
\end{aligned}
$$

We need to evaluate the expectation $E\left(K\left(n^{\alpha} S_{j}^{H_{1}}\right) K\left(n^{\alpha} S_{k}^{H_{1}}\right)\right)$. Let $\Xi$ be the covariance matrix of $\left(S_{j}^{H_{1}}, S_{k}^{H_{1}}\right)$ given by

$$
\Xi=\left(\begin{array}{cc}
\beta_{H_{1}} j^{2 H_{1}} & C_{H_{1}}(j, k) \\
C_{H_{1}}(j, k) & \beta_{H_{1}} k^{2 H_{1}}
\end{array}\right) .
$$


We have $|\Xi|=\beta_{H_{1}}^{2}(j k)^{2 H_{1}}-C_{H_{1}}^{2}(j, k)$ and

$$
\Xi^{-1}=\frac{1}{|\Xi|}\left(\begin{array}{cc}
\beta_{H_{1}} k^{2 H_{1}} & -C_{H_{1}}(j, k) \\
-C_{H_{1}}(j, k) & \beta_{H_{1}} j^{2 H_{1}}
\end{array}\right) \text {. }
$$

The density of $\left(S_{j}^{H_{1}}, S_{k}^{H_{1}}\right)$ is then

$$
g(x, y)
$$

$$
\begin{aligned}
& =\frac{1}{2 \pi \sqrt{|\Xi|}} \exp \left\{-\frac{1}{2|\Xi|}\left(\beta_{H_{1}} k^{2 H_{1}} x^{2}-2 C_{H_{1}}(j, k) x y\right.\right. \\
& \left.\left.+\beta_{H_{1}} j^{2 H_{1}} y^{2}\right)\right\} \text {. }
\end{aligned}
$$

We obtain

$$
\begin{aligned}
& E\left(K\left(n^{\alpha} S_{j}^{H_{1}}\right) K\left(n^{\alpha} S_{k}^{H_{1}}\right)\right) \\
& =\frac{1}{(2 \pi)^{2}|\Xi|^{1 / 2}} \int_{\mathbb{R}^{2}} e^{-\left(n^{2 \alpha} x^{2} / 2\right)} e^{-\left(n^{2 \alpha} y^{2} / 2\right)} g(x, y) d x d y \\
& =\frac{1}{(2 \pi)^{2}|\Xi|^{1 / 2}} \int_{\mathbb{R}} e^{-\left(n^{2 \alpha} y^{2} / 2\right)} e^{-\left(\beta_{H_{1}} j^{2 H_{1}} y^{2} / 2|\Xi|\right)} \int_{\mathbb{R}} e^{-\left(n^{2 \alpha} x^{2} / 2\right)} e^{-(1 / 2|\Xi|)\left(\beta_{H_{1}} k^{2 H_{1}} x^{2}-2 C_{H_{1}}(j, k) x y\right)} d x d y \\
& =\frac{1}{(2 \pi)^{2}|\Xi|^{1 / 2}} \int_{\mathbb{R}} e^{-\left(y^{2} / 2\right)\left(n^{2 \alpha}+\beta_{H_{1}} j^{2 H_{1}} /|\Xi|\right)} \int_{\mathbb{R}} e^{-1 / 2}\left[x^{2}\left(n^{2 \alpha}+\frac{\beta_{H_{1}} k^{2 H_{1}}}{|\Xi|}\right)-\frac{2 C_{H_{1}}(j, k)}{|\Xi|}\right] d x d y \\
& =\frac{1}{(2 \pi)^{2}|\Xi|^{1 / 2}} \int_{\mathbb{R}} e^{-\left(y^{2} / 2\right)\left(n^{2 \alpha}+\beta_{H_{1}} j^{2 H_{1}} /|\Xi|\right)} e^{-\left(\left(n^{2 \alpha}+\beta_{H_{1}} k^{2 H_{1}} /|\Xi|\right) / 2\right) \cdot\left(C_{H_{1}}^{2}(j, k) y^{2} /\left(n^{2 \alpha}+\beta_{H_{1}} k^{2 H_{1}} /|\Xi|\right)^{2}|\Xi|^{2}\right)} \\
& \cdot \int_{\mathbb{R}} e^{-\left(\left(n^{2 \alpha}+\beta_{H_{1}} k^{2 H_{1}} /|\Xi|\right) / 2\right)\left(x-C_{H_{1}}(j, k) y / n^{2 \alpha}|\Xi|+\beta_{H_{1}} k^{2 H_{1}}\right)^{2}} d x d y \\
& =\frac{1}{(2 \pi)^{3 / 2}|\Xi|^{1 / 2}} \cdot \frac{1}{\sqrt{n^{2 \alpha}+\beta_{H_{1}} k^{2 H_{1} /|\Xi|}}} \int_{\mathbb{R}} e^{-\left(y^{2} / 2\right)\left(\left(\left(|\Xi| n^{2 \alpha}+\beta_{H_{1}} j^{2 H_{1}}\right)\left(|\Xi| n^{2 \alpha}+\beta_{H_{1}} k^{2 H_{1}}\right)-C_{H_{1}}^{2}(j, k)\right) /|\Xi|\left(|\Xi| n^{2 \alpha}+\beta_{H_{1}} k^{2 H_{1}}\right)\right)} d y \\
& =\frac{1}{2 \pi|\Xi|^{1 / 2}} \cdot \frac{1}{\sqrt{n^{2 \alpha}+\beta_{H_{1}} k^{2 H_{1}} /|\Xi|}}\left(\frac{\left(|\Xi| n^{2 \alpha}+\beta_{H_{1}} j^{2 H_{1}}\right)\left(|\Xi| n^{2 \alpha}+\beta_{H_{1}} k^{2 H_{1}}\right)-C_{H_{1}}^{2}(j, k)}{|\Xi|\left(|\Xi| n^{2 \alpha}+\beta_{H_{1}} k^{2 H_{1}}\right)}\right)^{-1 / 2} .
\end{aligned}
$$

Thus

$$
\begin{aligned}
E( & \left.K\left(n^{\alpha} S_{j}^{H_{1}}\right) K\left(n^{\alpha} S_{k}^{H_{1}}\right)\right) \\
= & \frac{\sqrt{|\Xi|}}{2 \pi} \\
& \cdot \frac{1}{\sqrt{\left(|\Xi| n^{2 \alpha}+\beta_{H_{1}} j^{2 H_{1}}\right)\left(|\Xi| n^{2 \alpha}+\beta_{H_{1}} k^{2 H_{1}}\right)-C_{H_{1}}^{2}(j, k)}} \\
= & \frac{1}{2 \pi} \frac{1}{\sqrt{n^{4 \alpha}|\Xi|+\beta_{H_{1}} n^{2 \alpha}\left(j^{2 H_{1}}+k^{2 H_{1}}\right)+1}} .
\end{aligned}
$$

Suppose $j>k$; we use Lemma 1 to bound $|\Xi|=\beta_{H_{1}}^{2}(j k)^{2 H_{1}}-$ $C_{H_{1}}^{2}(j, k)$ from below; therefore

$$
\begin{aligned}
& E\left(K\left(n^{\alpha} S_{j}^{H_{1}}\right) K\left(n^{\alpha} S_{k}^{H_{1}}\right)\right) \\
& \quad \leq \frac{1}{2 \pi \sqrt{n^{4 \alpha} C_{H_{1}}(j-k)^{2 H_{1}} k^{2 H_{1}}+\beta_{H_{1}} n^{2 \alpha}\left(j^{2 H_{1}}+k^{2 H_{1}}\right)}} .
\end{aligned}
$$

By virtue of classical inequality $a^{2}+b^{2} \geq 2 a b$ with $a^{2}=$ $n^{4 \alpha} C_{H_{1}}(j-k)^{2 H_{1}} k^{2 H_{1}}$ and $b^{2}=\beta_{H_{1}} n^{2 \alpha}\left(j^{2 H_{1}}+k^{2 H_{1}}\right)$,

$$
\begin{aligned}
& E\left(K\left(n^{\alpha} S_{j}^{H_{1}}\right) K\left(n^{\alpha} S_{k}^{H_{1}}\right)\right) \\
& \quad \leq \frac{n^{-(3 / 2) \alpha}}{2 \pi 2^{3 / 4}\left(C_{H_{1}} \beta_{H_{1}}\right)^{1 / 4}(j-k)^{H_{1} / 2} j^{H_{1} / 4} k^{3 H_{1} / 4}} .
\end{aligned}
$$

For the function $f_{H_{2}}(j, k)$, according to [17], we can rewrite it as follows:

$$
\begin{aligned}
f_{H_{2}}(j, k)= & H_{2}\left(2 H_{2}-1\right)\left(2-2 H_{2}\right) \\
& \times \int_{0}^{1} \int_{0}^{1} \int_{-k}^{k+1}(j-1+x+y+z)^{2 H_{2}-3} d x d y d z,
\end{aligned}
$$


and thus

$$
\begin{aligned}
& \sum_{j>k} \int_{0}^{1} \int_{0}^{1} \int_{-k}^{k+1}(j-1+x+y+z)^{2 H_{2}-3} d x d y d z \\
& \quad=\int_{0}^{1} \int_{0}^{1} \int_{-k}^{k+1} \sum_{j>k}(j-1+x+y+z)^{2 H_{2}-3} d x d y d z \\
& \quad \leq \sum_{j>k} C(j-k)^{3-2 H_{2}}
\end{aligned}
$$

So

$$
\begin{aligned}
B \leq C \sum_{j>k}^{n-1} \frac{n^{-(3 / 2) \alpha}}{2} & \frac{2^{3 / 4}\left(C_{H_{1}} \beta_{H_{1}}\right)^{1 / 4}(j-k)^{H_{1} / 2} j^{H_{1} / 4} k^{3 H_{1} / 4}}{} \\
& \times(j-k)^{3-2 H_{2}},
\end{aligned}
$$

and consequently

$$
\begin{aligned}
B \leq & \frac{C n^{-(3 / 2) \alpha-(3 / 2) H_{1}+2 H_{2}-1}}{2 \pi 2^{3 / 4}\left(C_{H_{1}} \beta_{H_{1}}\right)^{1 / 4}} \\
& \times \underbrace{\sum_{j>k}^{n-1} \frac{1}{n^{2}} \frac{((j-k) / n)^{-\left(H_{1} / 2\right)+2 H_{2}-3}}{(j / n)^{H_{1} / 4}(k / n)^{3 H_{1} / 4}}}_{\rightarrow C\left(H_{1}, H_{2}\right)>0 \text { as } n \rightarrow \infty} \\
& \lesssim \frac{C\left(H_{1}, H_{2}\right)}{2 \pi 2^{3 / 4}\left(C_{H_{1}} \beta_{H_{1}}\right)^{1 / 4}} n^{-(3 / 2) \alpha-(3 / 2) H_{1}+2 H_{2}-1} .
\end{aligned}
$$

It follows that, under condition (47), $n^{\alpha+H_{1}-1} B$ converges to zero as $n$ tends to infinity.

As a consequence of Propositions 4 and 5 we obtain the following $L^{2}$-norm estimate for $S_{n}$.

Theorem 6. Suppose condition (47) holds; then, as $n$ tends to infinity,

$$
\begin{aligned}
\frac{\left[\beta_{H_{2}} \wedge 1\right]}{2 \pi \sqrt{2 \beta_{H_{2}}}\left(1-H_{1}\right)} & \leq \lim _{n \rightarrow \infty} n^{\alpha+H_{1}-1} E\left(S_{n}\right)^{2} \\
& \leq \frac{\left[\beta_{H_{2}} \vee 1\right]}{2 \pi \sqrt{2 \beta_{H_{2}}}\left(1-H_{1}\right)}
\end{aligned}
$$

\section{Limit Distribution of $\langle S\rangle_{n}$}

Theorem 6 implies that the diagonal part of $S_{n}^{2}$ is dominant in relation to the non-diagonal part, in the sense that this diagonal part is responsible for the renormalization order of $S_{n}^{2}$ which is $n^{\alpha+H_{1}-1}$. As a consequence we need to investigate the limit distribution of

$$
n^{\alpha+\mathrm{H}_{1}-1}\langle S\rangle_{n}=n^{\alpha+H_{1}-1} \sum_{j=0}^{n-1} K^{2}\left(n^{\alpha} S_{j}^{H_{1}}\right) .
$$

Using the self-similarity of sub-fractional Brownian motion, we have

$$
\begin{aligned}
& n^{\alpha+H_{1}-1} \sum_{j=0}^{n-1} K^{2}\left(n^{\alpha} S_{j}^{H_{1}}\right) \\
& =n^{\alpha+H_{1}-1} \sum_{j=0}^{n-1} K^{2}\left(n^{\alpha+H_{1}} S_{j / n}^{H_{1}}\right) .
\end{aligned}
$$

The limit of sequence (64) is linked to the local time of the sub-fractional Brownian motion $S^{H_{1}}$. From Geman-Horwitz one can find that the sub-fractional Brownian motion $S^{H_{1}}$ has a local time $\mathscr{L}^{H_{1}}(t, x)$ continuous in $(t, x) \in[0, \infty) \times \mathbb{R}$ which satisfies the occupation formula

$$
\int_{0}^{t} \phi\left(S_{s}^{H_{1}}, s\right) d s=\int_{\mathbb{R}} d x \phi(s, x) \mathscr{L}^{H_{1}}(d s, x)
$$

for every continuous and bounded function $\phi(t, x):[0, \infty) \times$ $\mathbb{R} \mapsto \mathbb{R}$ and such that

$$
\begin{aligned}
\mathscr{L}^{H_{1}}(t, x) & =\int_{0}^{t} \delta\left(S_{s}^{H_{1}}-x\right) d s \\
& =\lim _{\varepsilon \downarrow 0} \lambda\left(s \in[0, t],\left|S_{s}^{H}-x\right|<\varepsilon\right),
\end{aligned}
$$

where $\lambda$ denotes the Lebesgue measure and $\delta(x)$ is the Dirac delta function.

Next we will give an important convergence result that will be necessary in proving the main result of this section.

Proposition 7. The following convergence in distribution result holds:

$$
\begin{aligned}
\lim _{n \rightarrow \infty} n^{\alpha+H_{1}}( & \frac{1}{n} \sum_{j=0}^{n-1} K^{2}\left(n^{\alpha+H_{1}} S_{j / n}^{H_{1}}\right) \\
& \left.-\int_{0}^{t} K^{2}\left(n^{\alpha+H_{1}} S_{s}^{H_{1}}\right) d s\right)=0 .
\end{aligned}
$$

Proof. For fixed $\varepsilon>0$, denote by $p_{\varepsilon}(x)$ the Gaussian kernel with variance $\varepsilon>0$ given by $p_{\varepsilon}(x)=(1 / \sqrt{2 \pi \varepsilon}) e^{-x^{2} / 2 \varepsilon}$. Note that for every $s>0$

$$
\sqrt{\pi} n^{\alpha+H_{1}} K^{2}\left(n^{\alpha+H_{1}} S_{s}^{H_{1}}\right)=\frac{1}{2} p_{1 / 2 n^{2\left(\alpha+H_{1}\right)}}\left(S_{s}^{H_{1}}\right) .
$$


Using (68) we can rewrite the left-hand side of (67) as

$$
\begin{aligned}
\sqrt{\pi} & n^{\alpha+H_{1}}\left(\int_{0}^{1} K^{2}\left(n^{\alpha+H_{1}} S_{s}^{H_{1}}\right) d s-\frac{1}{n} \sum_{j=0}^{n-1} K^{2}\left(n^{\alpha+H_{1}} S_{j / n}^{H_{1}}\right)\right) \\
= & \frac{1}{2}\left[\int_{0}^{1} p_{1 / 2 n^{2\left(\alpha+H_{1}\right)}}\left(S_{s}^{H_{1}}\right) d s-\frac{1}{n} \sum_{j=0}^{n-1} p_{1 / 2 n^{2\left(\alpha+H_{1}\right)}}\left(S_{j / n}^{H_{1}}\right)\right] \\
= & \frac{1}{2} \sum_{j=0}^{n-1}\left[\int _ { j / n } ^ { ( j + 1 ) / n } \left(p_{1 / 2 n^{2\left(\alpha+H_{1}\right)}}\left(S_{s}^{H_{1}}\right)\right.\right. \\
= & \frac{1}{2} \sum_{j=0}^{n-1} \int_{j / n}^{(j+1) / n}\left(p_{1 / 2 n^{2\left(\alpha+H_{1}\right)}}\left(S_{s}^{H_{1}}\right)-p_{\varepsilon}\left(S_{s}^{H_{1}}\right)\right) d s \\
& +\frac{1}{2} \sum_{j=0}^{n-1} \int_{j / n}^{(j+1) / n}\left(p_{\varepsilon}\left(S_{s}^{H_{1}}\right)-p_{\varepsilon}\left(S_{j / n}^{H_{1}}\right)\right) d s \\
& \left.\left.+\frac{1}{2} \sum_{j=0}^{n-1} \int_{j / n}^{(j+1) / n}\left(S_{j / n}^{H_{1}}\right)\right) d s\right] \\
& \frac{1}{2}\left(I_{n}^{1}+I_{n}^{2}+I_{n}^{3}\right) .
\end{aligned}
$$

We will show that each of $I_{n}^{i}, i=1,2,3$, converges to zero (in some sense). Let us first consider the term $I_{n}^{1}$. We have

$$
I_{n}^{1}=\int_{0}^{1}\left(p_{1 / 2 n^{2\left(\alpha+H_{1}\right)}}\left(s_{s}^{H_{1}}\right)-p_{\varepsilon}\left(S_{s}^{H_{1}}\right)\right) d s .
$$

It follows from Yan et al. [9] that

$$
\lim _{\varepsilon \downarrow 0} \int_{0}^{1} p_{\varepsilon}\left(s_{s}^{H_{1}}\right) d s=\int_{0}^{1} \delta\left(S_{s}^{H_{1}}\right) d s=\mathscr{L}^{H_{1}}(1,0),
$$

in $L^{2}$ and almost surely, where $\mathscr{L}^{H_{1}}(1,0)$ is the local time of sub-fractional Brownian motion. Then $I_{n}^{1}$ tends to zero as $\varepsilon \rightarrow 0$ and $n \rightarrow \infty$. For the second term $I_{n}^{2}$ one can express it as follows:

$$
I_{n}^{2}=-\frac{1}{n} \sum_{j=0}^{n-1} p_{\varepsilon}\left(S_{j / n}^{H_{1}}\right)+\int_{0}^{1} p_{\varepsilon}\left(S_{s}^{H_{1}}\right) d s,
$$

and for every $\varepsilon>0$ it converges almost surely to zero as $n$ tends to infinity using the convergence of Riemann sum convergence. Let us now handle the term $I_{n}^{3}$ given by

$$
I_{n}^{3}=\frac{1}{n} \sum_{j=0}^{n-1}\left(p_{\varepsilon}\left(S_{j / n}^{H_{1}}\right)-p_{1 / 2 n^{2\left(\alpha+H_{1}\right)}}\left(S_{j / n}^{H_{1}}\right)\right) .
$$

We will treat $I_{n}^{3}$ by using the chaos decomposition of the Gaussian kernel applied to random variables in the first
Wiener chaos. Recall that for every $\varphi \in \mathscr{H}_{H_{1}}$ (which is the canonical Hilbert space associated with the sub-fractional Brownian motion $S^{H_{1}}$ )

$$
p_{\varepsilon}\left(S^{H_{1}}(\varphi)\right)=\sum_{m \geq 0} C_{m} I_{2 m}\left(\varphi^{\otimes 2 m}\right) \frac{1}{\left(\|\varphi\|_{\mathscr{H}_{H_{1}}}+\varepsilon\right)^{m+1 / 2}},
$$

where $C_{m}=(-1)^{m} / \sqrt{2 \pi} 2^{m} m$ !. Using the chaos decomposition we can write $p_{\varepsilon}\left(S_{j / n}^{H_{1}}\right)-p_{1 / 2 n^{2\left(\alpha+H_{1}\right)}}\left(S_{j / n}^{H_{1}}\right)$ almost surely as follows:

$$
\begin{aligned}
& p_{\varepsilon}\left(S_{j / n}^{H_{1}}\right)-p_{1 / 2 n^{2\left(\alpha+H_{1}\right)}}\left(S_{j / n}^{H_{1}}\right) \\
& =\sum_{m \geq 0} C_{m} I_{2 m}\left(1_{[0, j / n]}^{\otimes 2 m}\right) \\
& \times\left(\frac{1}{\left(\beta_{H_{1}}(j / n)^{2 H_{1}}+\varepsilon\right)^{m+1 / 2}}\right) \\
& \left.-\frac{1}{\left(\beta_{H_{1}}(j / n)^{2 H_{1}}+1 / 2 n^{2\left(\alpha+H_{1}\right)}\right)^{m+1 / 2}}\right) \\
& =\sum_{m \geq 0} C_{m} I_{2 m}\left(1_{[0, j / n]}^{\otimes 2 m}\right)\left(\frac{j}{n}\right)^{-2 H_{1}(m+1 / 2)} \\
& \times \beta^{-(m+1 / 2)} d_{j, \varepsilon, n, m},
\end{aligned}
$$

where

$$
\begin{aligned}
d_{j, \varepsilon, n, m}= & \left(\frac{\beta_{H_{1}}(j / n)^{2 H_{1}}}{\beta_{H_{1}}(j / n)^{2 H_{1}}+\varepsilon}\right)^{m+1 / 2} \\
& -\left(\frac{\beta_{H_{1}}(j / n)^{2 H_{1}}}{\beta_{H_{1}}(j / n)^{2 H_{1}}+1 / 2 n^{2\left(\alpha+H_{1}\right)}}\right)^{m+1 / 2} .
\end{aligned}
$$

We will show that $I_{n}^{3}$ converges to zero in $L^{2}$ as $n$ tends to infinity and $\varepsilon$ tends to zero. From (73) we can easily see that the diagonal part of $I_{n}^{3}$ converges to zero. We can also see, from the expression of $I_{n}^{3}$, that the summands with $j=0$ vanish. Then, by using the orthogonality of multiple stochastic integral, we obtain

$$
\begin{aligned}
E\left(I_{n}^{3}\right)^{2}=\frac{1}{n^{2}} \sum_{m \geq 0} C_{m}^{2}(2 m) ! & \\
& \times \sum_{j, k \geq 1, j \neq k}^{n-1}\left\langle 1_{[0, j / n]}, 1_{[0, k / n]}\right\rangle_{\mathscr{H}_{H_{1}}}^{2 m} \beta_{H_{1}}^{-(2 m+1)} \\
& \cdot\left(\frac{j}{n} \cdot \frac{k}{n}\right)^{-2 H_{1}(m+1 / 2)} d_{j, \varepsilon, n, m} d_{k, \varepsilon, n, m} .
\end{aligned}
$$


We can also write

$$
\begin{aligned}
& E\left(I_{n}^{3}\right)^{2} \sim \frac{1}{n^{2}} \sum_{m \geq 0} C_{m}^{2}(2 m) ! \\
& \times \sum_{j, k \geq 1, j \neq k}^{n-1} C_{H_{1}}^{2 m}\left(\frac{j}{n}, \frac{k}{n}\right) \beta_{H_{1}}^{-(2 m+1)} \\
& \cdot\left(\frac{j}{n} \cdot \frac{k}{n}\right)^{-2 H_{1}(m+1 / 2)} d_{j, \varepsilon, n, m} d_{k, \varepsilon, n, m} \\
&: \equiv \sum_{m \geq 0} C_{m}^{2}(2 m) ! D_{m}(\varepsilon, n),
\end{aligned}
$$

where

$$
\begin{aligned}
D_{m}(\varepsilon, n)=\frac{1}{n^{2}} \sum_{j, k \geq 1, j \neq k}^{n-1} C_{H_{1}}^{2 m}\left(\frac{j}{n}, \frac{k}{n}\right) \beta_{H_{1}}^{-(2 m+1)} \\
\cdot\left(\frac{j}{n} \cdot \frac{k}{n}\right)^{-2 H_{1}(m+1 / 2)} d_{j, \varepsilon, n, m} d_{k, \varepsilon, n, m} .
\end{aligned}
$$

We now claim that, for every fixed $m \geq 1$,

$$
\lim _{\varepsilon \rightarrow 0} \lim _{n \rightarrow \infty} D_{m}(\varepsilon, n)=0 .
$$

In fact, for every $m \geq 0$, we get

$$
\begin{aligned}
\left|d_{j, \varepsilon, n, m}\right|= & \mid\left(\frac{\beta_{H_{1}}(j / n)^{2 H_{1}}}{\beta_{H_{1}}(j / n)^{2 H_{1}}+\varepsilon}\right)^{m+1 / 2}-1 \\
& +1-\left(\frac{\beta_{H_{1}}(j / n)^{2 H_{1}}}{\beta_{H_{1}}(j / n)^{2 H_{1}}+1 / 2 n^{2\left(\alpha+H_{1}\right)}}\right)^{m+1 / 2} \mid \\
\leq & \left|1-\left(\frac{\beta_{H_{1}}(j / n)^{2 H_{1}}}{\beta_{H_{1}}(j / n)^{2 H_{1}}+\varepsilon}\right)^{m+1 / 2}\right| \\
& +\left|1-\left(\frac{\beta_{H_{1}}(j / n)^{2 H_{1}}}{\beta_{H_{1}}(j / n)^{2 H_{1}}+1 / 2 n^{2\left(\alpha+H_{1}\right)}}\right)^{m+1 / 2}\right| \\
\leq & \left|1-\left(\frac{\beta_{H_{1}}(j / n)^{2 H_{1}}}{\beta_{H_{1}}(j / n)^{2 H_{1}}+\varepsilon}\right)^{m+1}\right| \\
& +\left|1-\left(\frac{\beta_{H_{1}}(j / n)^{2 H_{1}}}{\beta_{H_{1}}(j / n)^{2 H_{1}}+1 / 2 n^{2\left(\alpha+H_{1}\right)}}\right)^{m+1}\right| \\
= & c_{m}\left(\left|\left(\frac{\varepsilon}{\beta_{H_{1}}(j / n)^{2 H_{1}}+\varepsilon}\right)\right|\right. \\
& +\left|\left(\frac{1 / 2 n^{2\left(\alpha+H_{1}\right)}}{\beta_{H_{1}}(j / n)^{2 H_{1}}+1 / 2 n^{2\left(\alpha+H_{1}\right)}}\right)\right|
\end{aligned}
$$

Now for every $j, n, m$ we have $\lim _{\varepsilon \rightarrow 0}\left|\operatorname{frac} \varepsilon \beta_{H_{1}}(j / n)^{2 H_{1}}+\varepsilon\right|=$ 0 for every $j \geq 1$ and

$$
\begin{aligned}
& \frac{1 / 2 n^{2\left(\alpha+H_{1}\right)}}{\beta_{H_{1}}(j / n)^{2 H_{1}}+1 / 2 n^{2\left(\alpha+H_{1}\right)}} \\
& \leq \frac{1 / 2 n^{2\left(\alpha+H_{1}\right)}}{\beta_{H_{1}}(1 / n)^{2 H_{1}}+1 / 2 n^{2\left(\alpha+H_{1}\right)}} \longrightarrow 0,
\end{aligned}
$$

as $n$ tends to infinity because of $\alpha>0$.

Furthermore, we have that

$$
\frac{1}{n^{2}} \sum_{j, k \geq 1}^{n-1} C_{H_{1}}^{2 m}\left(\frac{j}{n}, \frac{k}{n}\right) \beta_{H_{1}}^{-(2 m+1)} \cdot\left(\frac{j}{n} \cdot \frac{k}{n}\right)^{-2 H_{1}(m+1 / 2)}
$$

converges to

$$
\beta_{H_{1}}^{-(2 m+1)} \int_{0}^{1} \int_{0}^{1} C_{H_{1}}^{2 m}(u, v)(u v)^{-2 H_{1}(m+1 / 2)}
$$

as $n$ tends to infinity. Since the quantity is finite, it implies (80).

We will now prove

$$
\sum_{m \geq 0} C_{m}^{2}(2 m) ! \sup _{\varepsilon, n}\left|D_{m}(\varepsilon, n)\right|<\infty .
$$

Equalities (80) and (85) will imply the convergence of $I_{n}^{3}$ to zero in $L^{2}$. Furthermore one can easily check

$$
\left|d_{j, \varepsilon, n, m}\right| \leq 1, \quad\left|d_{k, \varepsilon, n, m}\right| \leq 1
$$

By bounding from above the terms $\left|d_{j, \varepsilon, n, m}\right|$ and $\left|d_{k, \varepsilon, n, m}\right|$ by 1 in $\sum_{m \geq 0} C_{m}^{2}(2 m) ! \sup _{\varepsilon, n}\left|D_{m}(\varepsilon, n)\right|$, we obtain that

$$
\begin{gathered}
\sum_{m \geq 0} C_{m}^{2}(2 m) ! \sup _{\varepsilon, n}\left|D_{m}(\varepsilon, n)\right| \\
\leq \sum_{m \geq 0} C_{m}^{2}(2 m) ! \sup _{n} \frac{1}{n^{2}} \\
\cdot \sum_{j, k \geq 1, j \neq k}^{n-1} C_{H_{1}}^{2 m}\left(\frac{j}{n}, \frac{k}{n}\right) \beta_{H_{1}}^{-(2 m+1)} \\
\cdot\left(\frac{j}{n} \cdot \frac{k}{n}\right)^{-2 H_{1}(m+1 / 2)} \\
=\sum_{m \geq 0} C_{m}^{2}(2 m) ! \sup _{n} \frac{1}{n^{2}} C_{H_{1}}^{2 m}\left(1, \frac{k}{j}\right) \\
\quad \times \beta_{H_{1}}^{-(2 m+1)}\left(\frac{k}{j}\right)^{-2 m H_{1}}\left(\frac{j}{n} \cdot \frac{k}{n}\right)^{-H_{1}} .
\end{gathered}
$$


Let us focus on the case where $H<1 / 2$ first. For the function $Q_{H_{1}}(z)$ defined by (31), due to Yan and Shen [8], one can check $Q_{H_{1}}(z) \leq \beta_{H_{1}} z^{H_{1}}$. It follows that

$$
\begin{aligned}
& \sum_{m \geq 0} C_{m}^{2}(2 m) ! \sup _{\varepsilon, n}\left|D_{m}(\varepsilon, n)\right| \\
& \leq 2 \sum_{m \geq 0} C_{m}^{2}(2 m) ! \sup _{n} \frac{1}{n^{2}} \\
& \times \sum_{j, k \geq 0, j>k}^{n-1}\left(\frac{k}{j}\right)^{2 m H_{1}} \beta_{H_{1}}^{-1}\left(\frac{j}{n} \cdot \frac{k}{n}\right)^{-H_{1}} \\
& \leq 2 \sum_{m \geq 0} C_{m}^{2}(2 m) ! \sup _{n} \frac{1}{n^{2}} \\
& \times \sum_{j, k \geq 1, j>k}^{n-1} \beta_{H_{1}}^{-1}\left(\frac{k}{n}\right)^{H_{1}(2 m-1)}\left(\frac{j}{n}\right)^{-H_{1}(2 m+1)} \\
& \leq 2 \sum_{m \geq 0} C_{m}^{2}(2 m) ! \sup _{n} \frac{1}{n^{2-2 H_{1}}} \\
& \times \sum_{j=0}^{n-1} j^{-H_{1}(2 m+1)} \beta_{H_{1}}^{-1} \\
& \quad \times \sum_{k=0}^{j-1} \int_{k}^{k+1} k^{H_{1}(2 m-1)} d x
\end{aligned}
$$$$
\leq 2 \sum_{m \geq 0} C_{m}^{2}(2 m) ! \sup _{n} \frac{1}{n^{2-2 H_{1}}}
$$$$
\times \sum_{j=0}^{n-1} j^{-H_{1}(2 m+1)} \beta_{H_{1}}^{-1} \int_{0}^{j} x^{H_{1}(2 m-1)} d x
$$$$
\leq 2 \sum_{m \geq 0} C_{m}^{2}(2 m) ! \sup _{n} \frac{1}{n^{2-2 H_{1}}}
$$$$
\times \frac{\beta_{H_{1}}^{-1}}{H_{1}(2 m-1)+1} \sum_{j=0}^{n-1} j^{1-2 H_{1}}
$$$$
\leq 2 \sum_{m \geq 0} C_{m}^{2}(2 m) ! \sup _{n} \frac{1}{n^{2-2 H_{1}}}
$$

$$
\begin{gathered}
\times \frac{\beta_{H_{1}}^{-1}}{H_{1}(2 m-1)+1} \sum_{j=0}^{n-1} n^{1-2 H_{1}} \\
=2 \sum_{m \geq 0} C_{m}^{2}(2 m) ! \sup _{n} \frac{\beta_{H_{1}}^{-1}}{H_{1}(2 m-1)+1}
\end{gathered}
$$

Given that, by using Stirling's formula, the coefficient $C_{m}^{2}(2 m)$ ! behaves as $1 / \sqrt{m}$, we obtain that the above sum is finite. Thus we obtain the convergence of $I_{n}^{3}$ to zero in $L^{2}$ for $H_{1}<1 / 2$.

Let us now treat the case $H_{1}>1 / 2$. We can easily check that the function $Q_{H_{1}}$ defined by (31) is increasing on $[0,1]$.
Since $k / j \leq(j-1) / j=1-1 / j$, it holds that $Q_{H_{1}}(k / j) \leq$ $Q_{H_{1}}(1-1 / j)$. Then

$$
\begin{aligned}
& \sum_{m \geq 0} C_{m}^{2}(2 m) ! \sup _{\varepsilon, n}\left|D_{m}(\varepsilon, n)\right| \\
& \leq 2 \sum_{m \geq 0} C_{m}^{2}(2 m) ! \sup _{n} \frac{1}{n^{2}} \\
& \times \sum_{j=0}^{n-1} Q_{H_{1}}\left(1-\frac{1}{j}\right) \sum_{k=0}^{j-1}\left(\frac{j}{n} \cdot \frac{k}{n}\right)^{-H_{1}} \beta_{H_{1}}^{-(2 m+1)} \\
& \leq 2 \sum_{m \geq 0} C_{m}^{2}(2 m) ! \beta_{H_{1}}^{-(2 m+1)} \sup _{n} \frac{1}{n} \\
& \quad \times \sum_{j=0}^{n-1} Q_{H_{1}}\left(1-\frac{1}{j}\right)\left(\frac{j}{n}\right)^{-H_{1}} \sum_{k=0}^{j-1} \int_{(k-1) / n}^{k / n} x^{-H_{1}} d x \\
& \leq C_{H} \sum_{m \geq 0} C_{m}^{2}(2 m) ! \beta_{H_{1}}^{-(2 m+1)} \sup _{n} \frac{1}{n} \\
& \quad \times \sum_{j=0}^{n-1} Q_{H_{1}}\left(1-\frac{1}{j}\right)\left(\frac{j}{n}\right)^{-H_{1}}\left(\frac{j-1}{n}\right)^{1-H_{1}} \\
& \sim C_{H} \sum_{m \geq 0} C_{m}^{2}(2 m) ! \beta_{H_{1}}^{-(2 m+1)} \sup _{n} \frac{1}{n} \\
& \quad \times \sum_{j=0}^{n-1} Q_{H_{1}}\left(1-\frac{1}{j}\right)\left(\frac{j}{n}\right)^{1-2 H_{1}} \cdot
\end{aligned}
$$

We know that $C_{H_{1}}(1, z) \leq \beta_{H_{1}} R_{H_{1}}(1, z)$; by adapting Lemma 2 in [18], we can prove that

$$
\begin{aligned}
\frac{1}{n} C_{H_{1}}^{2 m}\left(1, \frac{k}{j}\right) \beta_{H_{1}}^{-(2 m+1)}\left(\frac{k}{j}\right)^{-2 m H_{1}}\left(\frac{j}{n} \cdot \frac{k}{n}\right)^{-H_{1}} \\
\leq \frac{1}{n} R_{H_{1}}^{2 m}\left(1, \frac{k}{j}\right) \beta_{H_{1}}^{-1}\left(\frac{k}{j}\right)^{-2 m H_{1}}\left(\frac{j}{n} \cdot \frac{k}{n}\right)^{-H_{1}} \\
\leq C_{H_{1}} \frac{\beta_{H_{1}}^{-1}}{m^{1 / 2 H_{1}}}
\end{aligned}
$$

with $C_{H_{1}}$ depending only on $H_{1}$. As a consequence

$$
\sum_{m \geq 0} C_{m}^{2}(2 m) ! \sup _{\varepsilon, n}\left|D_{m}(\varepsilon, n)\right| \leq C_{H_{1}} C_{m}^{2}(2 m) ! \frac{\beta_{H_{1}}^{-1}}{m^{1 / 2 H_{1}}} .
$$

The Stirling formula implies again that the above series is convergent.

Theorem 8. Let $\langle S\rangle_{n}$ be defined by (63); then as $n$ tends to infinity, one has the convergence in distribution of

$$
\lim _{n \rightarrow \infty} n^{\alpha+H_{1}-1}\langle S\rangle_{n}=\int_{\mathbb{R}} K^{2}(y) d y \mathscr{L}^{H_{1}}(1,0),
$$

where $\mathscr{L}^{H_{1}}(1,0)$ is the local time of sub-fractional Brownian motion $S^{H_{1}}$. 
Proof. Using Proposition 7, it suffices to check that

$$
n^{\alpha+H_{1}} \int_{0}^{1} K^{2}\left(n^{\alpha+H_{1}} S_{s}^{H_{1}}\right) d s \longrightarrow \int_{\mathbb{R}} K^{2}(y) d y \mathscr{L}^{H_{1}}(1,0),
$$

as $n$ tends to infinity. Using the occupation time formula, one can obtain

$$
\begin{aligned}
n^{\alpha+H_{1}} \int_{0}^{1} & K^{2}\left(n^{\alpha+H_{1}} S_{s}^{H_{1}}\right) d s \\
& =n^{\alpha+H_{1}} \int_{\mathbb{R}} K^{2}\left(n^{\alpha+H_{1}} x\right) \mathscr{L}^{H_{1}}(1, d x) \\
& =\int_{\mathbb{R}} K^{2}(y) \mathscr{L}^{H_{1}}\left(1, y n^{-\alpha-H_{1}}\right) d y,
\end{aligned}
$$

which converges to $\int_{\mathbb{R}} K^{2}(y) d y \mathscr{L}^{H_{1}}(1,0)$ as $n$ tends to infinity by using the continuity of the local time $\mathscr{L}^{H_{1}}(t, x)$.

\section{Limit Distribution of $S_{n}$}

In this section, we will investigate the limit in distribution of

$$
S_{n}=\sum_{j=0}^{n-1} K\left(n^{\alpha} S_{j}^{H_{1}}\right)\left(S_{j+1}^{H_{2}}-S_{j}^{H_{2}}\right)
$$

Let us consider the Gaussian vector

$$
X^{H_{2}}=\left(X_{1}^{H_{2}}, \ldots, X_{n}^{H_{2}}\right)=\left(S_{1}^{H_{2}}-S_{0}^{H_{2}}, \ldots, S_{n}^{H_{2}}-S_{n-1}^{H_{2}}\right) \text {. }
$$

From the above definition, one can obtain

$$
\begin{aligned}
S_{n} & =\sum_{j=0}^{n-1} K\left(n^{\alpha} S_{j}^{H_{1}}\right)\left(S_{j+1}^{H_{2}}-S_{j}^{H_{2}}\right) \\
& =\sum_{j=0}^{n-1} K\left(n^{\alpha} S_{j}^{H_{1}}\right) X_{j+1}^{H_{2}} .
\end{aligned}
$$

Theorem 9. Let $S_{n}$ be given by (95) and assume that

$$
\alpha<1-H_{1} \text {. }
$$

Then one has the convergence in law

$$
n^{\alpha+H_{1}-1} S_{n} \longrightarrow \int_{\mathbb{R}} K^{2}(y) d y W_{\mathscr{L}^{H_{1}}(t, 0)},
$$

where $\mathscr{L}^{H_{1}}(t, 0)$ is the local time of $S^{H_{1}}$ and $W$ is a Brownian motion independent of $S^{H_{1}}$.

Proof. We will study the characteristic function of $n^{\left(\alpha+H_{1}-1\right) / 2} S_{n}$. Let $i$ be the imaginary unit and $\lambda_{n}$ given by

$$
\lambda_{n}=\lambda n^{\left(\alpha+H_{1}-1\right) / 2}, \quad \lambda \in \mathbb{R} .
$$

Because of the independence of the two sub-fractional Brownian motions and computing the conditional expectation of $e^{i \lambda_{n}} S_{n}$ given $S^{H_{1}}$ we obtain

$$
E\left(e^{i \lambda_{n} S_{n}}\right)=E\left(e^{-(1 / 2) \sum_{j, k=0}^{n-1} \lambda_{n}^{2} K\left(n^{\alpha} S_{j}^{H_{1}}\right) K\left(n^{\alpha} S_{k}^{H_{1}}\right) f_{H_{2}}(j, k)}\right) .
$$

It follows that, with $g_{H_{2}}(j, k)$,

$$
\begin{aligned}
E\left(e^{i \lambda_{n} S_{n}}\right)= & E\left(e^{-(1 / 2) \lambda_{n}^{2} \sum_{j=0}^{n-1} K^{2}\left(n^{\alpha} S_{j}^{H_{1}}\right) f_{H_{2}}(j, j)} e^{-(1 / 2) \sum_{j \neq k=0}^{n-1} \lambda_{n}^{2} K\left(n^{\alpha} S_{j}^{H_{1}}\right) K\left(n^{\alpha} S_{k}^{H_{1}}\right) f_{H_{2}}(j, k)}\right) \\
= & E\left(e^{-(1 / 2) \lambda_{n}^{2} \sum_{j=0}^{n-1} K^{2}\left(n^{\alpha} S_{j}^{H_{1}}\right) f_{H_{2}}(j, j)} e^{-\sum_{j=0}^{n-1} \sum_{k=0}^{j-1} \lambda_{n}^{2} K\left(n^{\alpha} S_{j}^{H_{1}}\right) K\left(n^{\alpha} S_{k}^{H_{1}}\right) f_{H_{2}}(j, k)}\right) \\
= & E\left(e^{-(1 / 2) \lambda_{n}^{2} \sum_{j=0}^{n-1} K^{2}\left(n^{\alpha} S_{j}^{H_{1}}\right) f_{H_{2}}(j, j)} e^{-(1 / 2) \sum_{j=0}^{n-1} \sum_{k=0}^{j-1} \lambda_{n}^{2} K\left(n^{\alpha} S_{j}^{H_{1}}\right) K\left(n^{\alpha} S_{k}^{H_{1}}\right)}\right. \\
& \left.\cdot e^{-\lambda_{n}^{2} H_{2}\left(2 H_{2}-1\right) \int_{j}^{j+1} \int_{k}^{k+1}\left[(s-u)^{2 H_{2}-2}-(s+u)^{2 H_{2}-2}\right] d u d s}\right) \\
= & E\left(e^{-(1 / 2) \lambda_{n}^{2} \sum_{j=0}^{n-1} K^{2}\left(n^{\alpha} S_{j}^{H_{1}}\right) f_{H_{2}}(j, j)} \cdot e^{-\lambda_{n}^{2} H_{2}\left(2 H_{2}-1\right) \int_{0}^{n} \int_{0}^{[s]} K\left(n^{\alpha} S_{[s]}^{H_{1}}\right) K\left(n^{\alpha} S_{[u]}^{H_{1}}\right)\left[(s-u)^{2 H_{2}-2}-(s+u)^{2 H_{2}-2}\right] d u d s}\right) .
\end{aligned}
$$

Consider the process $V_{n}$ defined by

$$
\begin{aligned}
V_{n}=\int_{0}^{n} \int_{0}^{[s]} K\left(n^{\alpha} S_{[s]}^{H_{1}}\right) K\left(n^{\alpha} S_{[u]}^{H_{1}}\right) \\
\quad \times\left[(s-u)^{2 H_{2}-2}-(s+u)^{2 H_{2}-2}\right] d u d s
\end{aligned}
$$

and the function $h$ defined by $h(x)=e^{-\lambda_{n}^{2} H_{2}\left(2 H_{2}-1\right) x}$. Note that $V_{n}$ is a bounded variation process (its quadratic variation process is zero). Furthermore

$$
h^{\prime}(x)=h(x)\left(-\lambda_{n}^{2} H_{2}\left(2 H_{2}-1\right)\right) .
$$

Using the change of variables formula for bounded variation process it follows that

$$
h\left(V_{n}\right)=h(0)+\int_{0}^{n} h^{\prime}\left(V_{s}\right) d V_{s}
$$


which means that

$$
\begin{aligned}
h\left(V_{n}\right) & =e^{-\lambda_{n}^{2} H_{2}\left(2 H_{2}-1\right) V_{n}} \\
& =1-\lambda_{n}^{2} H_{2}\left(2 H_{2}-1\right) \int_{0}^{n} e^{-\lambda_{n}^{2} H_{2}\left(2 H_{2}-1\right) V_{s}} d V_{s} .
\end{aligned}
$$

Therefore

$$
\begin{aligned}
& E\left(e^{i \lambda_{n} S_{n}}\right) \\
& =E\left(e^{-(1 / 2) \lambda_{n}^{2} \sum_{j=0}^{n-1} K^{2}\left(n^{\alpha} S_{j}^{H_{1}}\right) f_{H_{2}}(j, j)}\right. \\
& \left.\quad \times\left(1-\lambda_{n}^{2} H_{2}\left(2 H_{2}-1\right) \int_{0}^{n} e^{-\lambda_{n}^{2} H_{2}\left(2 H_{2}-1\right) V_{s}} d V_{s}\right)\right) \\
& =E\left(e^{-(1 / 2) \lambda_{n}^{2} \sum_{j=0}^{n-1} K^{2}\left(n^{\alpha} S_{j}^{H_{1}}\right) f_{H_{2}}(j, j)}\right. \\
& \quad-\lambda_{n}^{2} H_{2}\left(2 H_{2}-1\right) e^{-(1 / 2) \lambda_{n}^{2} \sum_{j=0}^{n-1} K^{2}\left(n^{\alpha} S_{j}^{H_{1}}\right) g_{H_{2}}(j, j)} \\
& \left.\quad \times \int_{0}^{n} e^{-\lambda_{n}^{2} H_{2}\left(2 H_{2}-1\right) V_{s}} d V_{s}\right) \\
& : \equiv E\left(J_{n}^{(1)}\right)-E\left(J_{n}^{(2)}\right) .
\end{aligned}
$$

We will focus on the term $E\left(J_{n}^{(2)}\right)$ and show that

$$
\lim _{n \rightarrow \infty} E\left(\left|J_{n}^{(2)}\right|\right)=0
$$

From

$$
\begin{aligned}
d V_{s}=\left(\int_{0}^{[s]}\right. & K\left(n^{\alpha} S_{[s]}^{H_{1}}\right) K\left(n^{\alpha} S_{[u]}^{H_{1}}\right) \\
& \left.\times\left[(s-u)^{2 H_{2}-2}-(s+u)^{2 H_{2}-2}\right] d u\right) d s
\end{aligned}
$$

we obtain

$$
\begin{aligned}
& E\left(J_{n}^{(2)}\right) \\
& =E\left(\lambda_{n}^{2} H_{2}\left(2 H_{2}-1\right) e^{-(1 / 2) \lambda_{n}^{2} \sum_{j=0}^{n-1} K^{2}\left(n^{\alpha} S_{j}^{H_{1}}\right) f_{H_{2}}(j, j)}\right. \\
& \times \int_{0}^{n} e^{-\lambda_{n}^{2} H_{2}\left(2 H_{2}-1\right) V_{s}} \\
& \cdot \int_{0}^{[s]} K\left(n^{\alpha} S_{[s]}^{H_{1}}\right) K\left(n^{\alpha} S_{[u]}^{H_{1}}\right) \\
& \left.\times\left[(s-u)^{2 H_{2}-2}-(s+u)^{2 H_{2}-2}\right] d u d s\right)
\end{aligned}
$$

$$
\begin{aligned}
&=E\left(\lambda_{n}^{2} H_{2}\right.\left(2 H_{2}-1\right) \\
& \times \int_{0}^{n} e^{-(1 / 2) \lambda_{n}^{2} \int_{0}^{s} K^{2}\left(n^{\alpha} S_{[u]}^{H_{1}}\right) f_{H_{2}}(u, u) d u} \\
& \quad \times e^{-(1 / 2) \lambda_{n}^{2} \int_{s}^{n} K^{2}\left(n^{\alpha} S_{[u]}^{H_{1}}\right) f_{H_{2}}(u, u) d u} \\
& \cdot e^{-\lambda_{n}^{2} H_{2}\left(2 H_{2}-1\right) V_{s}} \\
& \quad \times \int_{0}^{[s]} K\left(n^{\alpha} S_{[s]}^{H_{1}}\right) K\left(n^{\alpha} S_{[u]}^{H_{1}}\right) \\
&\left.\quad \times\left[(s-u)^{2 H_{2}-2}-(s+u)^{2 H_{2}-2}\right] d u d s\right) .
\end{aligned}
$$

Recall that the following holds:

$$
\begin{aligned}
& E\left(e^{i \lambda_{n} S_{n}} \mid S_{s}^{H_{1}}\right) \\
& \quad=E\left(e^{-(1 / 2) \lambda_{n}^{2} \int_{0}^{s} K^{2}\left(n^{\alpha} S_{[u]}^{H_{1}}\right) f_{H_{2}}(u, u) d u} e^{-\lambda_{n}^{2} H_{2}\left(2 H_{2}-1\right) V_{s}} \mid S_{s}^{H_{1}}\right) .
\end{aligned}
$$

This can be seen for $s$ as an integer at the beginning of the proof and also it can be checked for any $s>0$. We will use this property to compute the following upper bound for $E\left(\left|J_{n}^{(2)}\right|\right)$ :

$$
\begin{aligned}
& E\left(\left|J_{n}^{(2)}\right|\right) \\
& \leq E\left(\lambda_{n}^{2} \int_{0}^{n} e^{-(1 / 2) \lambda_{n}^{2} \int_{0}^{s} K^{2}\left(n^{\alpha} S_{[u]}^{H_{1}}\right) f_{H_{2}}(u, u) d u}\right. \\
& \times\left|e^{-(1 / 2) \lambda_{n}^{2} \int_{s}^{n} K^{2}\left(n^{\alpha} S_{[u]}^{H_{1}}\right) f_{H_{2}}(u, u) d u}\right| \\
& \times e^{-\lambda_{n}^{2} H_{2}\left(2 H_{2}-1\right) V_{s}} \cdot H_{2}\left|2 H_{2}-1\right| \\
& \times \int_{0}^{[s]} K\left(n^{\alpha} S_{[s]}^{H_{1}}\right) K\left(n^{\alpha} S_{[u]}^{H_{1}}\right) \\
& \left.\times\left[(s-u)^{2 H_{2}-2}-(s+u)^{2 H_{2}-2}\right] d u d s\right) \\
& \leq E\left(\lambda _ { n } ^ { 2 } \int _ { 0 } ^ { n } E \left(e^{-(1 / 2) \lambda_{n}^{2} \int_{0}^{s} K^{2}\left(n^{\alpha} S_{[u]}^{H_{1}}\right) f_{H_{2}}(u, u) d u}\right.\right. \\
& \left.\times e^{-\lambda_{n}^{2} H_{2}\left(2 H_{2}-1\right) V_{s}} \mid S_{s}^{H_{1}}\right) \\
& \cdot H_{2}\left|2 H_{2}-1\right| \int_{0}^{[s]} K\left(n^{\alpha} S_{[s]}^{H_{1}}\right) K\left(n^{\alpha} S_{[u]}^{H_{1}}\right) \\
& \left.\times\left[(s-u)^{2 H_{2}-2}-(s+u)^{2 H_{2}-2}\right] d u d s\right) .
\end{aligned}
$$


This is because all the terms of the double integral are measurable with respect to the filtration generated by $\left(S_{r}^{H_{1}}, r \leq s\right)$. At this point we use (111) to write

$$
\begin{gathered}
E\left(\left|J_{n}^{(2)}\right|\right) \\
\leq E\left(\lambda_{n}^{2} \int_{0}^{n} E\left(e^{i \lambda_{n} S_{s}} \mid S_{s}^{H_{1}}\right) H_{2}\left|2 H_{2}-1\right|\right. \\
\cdot \int_{0}^{[s]} K\left(n^{\alpha} S_{[s]}^{H_{1}}\right) K\left(n^{\alpha} S_{[u]}^{H_{1}}\right) \\
\leq E\left(\lambda_{n}^{2} \int_{0}^{n}\left|e^{i \lambda_{n} S_{s}}\right| H_{2}\left|2 H_{2}-1\right|\right. \\
\quad \cdot \int_{0}^{[s]} K\left(n^{\alpha} S_{[s]}^{H_{1}}\right) K\left(n^{\alpha} S_{[u]}^{H_{1}}\right) \\
\leq E\left(\lambda_{n}^{2} \sum_{j=0}^{n-1} \sum_{k=0}^{j-1} K\left(n^{\alpha} S_{j}^{H_{1}}\right) K\left(n^{\alpha} S_{k}^{H_{1}}\right) H_{2}\left|2 H_{2}-1\right|\right. \\
\leq E\left(\lambda_{n}^{2} \int_{0}^{n} \int_{0}^{[s]} K\left(n^{\alpha} S_{[s]}^{H_{1}}\right) K\left(n^{\alpha} S_{[u]}^{H_{1}}\right) H_{2}\left|2 H_{2}-1\right|\right. \\
\left.\times\left[(s-u)^{2 H_{2}-2}\right] d u d s\right) \\
\left.\times\left[(s-u)^{2 H_{2}-2}-(s+u)^{2 H_{2}-2}\right] d u d s\right)
\end{gathered}
$$

Suppose that $\mathrm{H}_{2}>1 / 2$, which means that $2 \mathrm{H}_{2}-1>0$ and $g_{H_{2}}(j, k)>0$; consequently

$$
\begin{aligned}
& E\left(\left|J_{n}^{(2)}\right|\right) \\
& \quad \leq E\left(\frac{\lambda_{n}^{2}}{2} \sum_{j=0}^{n-1} \sum_{k=0}^{j-1} K\left(n^{\alpha} S_{j}^{H_{1}}\right) K\left(n^{\alpha} S_{k}^{H_{1}}\right) f_{H_{2}}(j, k)\right) \\
& \quad \leq E\left(\frac{\lambda^{2}}{2} n^{n+H_{1}-1} \sum_{j=0}^{n-1} \sum_{k=0}^{j-1} K\left(n^{\alpha} S_{j}^{H_{1}}\right) K\left(n^{\alpha} S_{k}^{H_{1}}\right) f_{H_{2}}(j, k)\right) .
\end{aligned}
$$

This term is exactly the nondiagonal term of the $L^{2}$-norm of $n^{\left(\alpha+H_{1}-1\right) / 2} S_{n}$ and we know that, under condition (98), it converges to zero as $n$ tends to infinity. Finally we have

$$
E\left(\left|J_{n}^{(2)}\right|\right) \longrightarrow 0, \quad \text { as } n \longrightarrow \infty
$$

Suppose $H_{2}<1 / 2$. It follows that $2 H_{2}-1<0, f_{H_{2}}(j, k)<0$, which gives us

$$
\begin{gathered}
E\left(\left|J_{n}^{(2)}\right|\right) \\
\leq E\left(-\frac{\lambda_{n}^{2}}{2} \sum_{j=0}^{n-1} \sum_{k=0}^{j-1} K\left(n^{\alpha} S_{j}^{H_{1}}\right)\right. \\
\left.\times K\left(n^{\alpha} S_{k}^{H_{1}}\right) f_{H_{2}}(j, k)\right) \\
\leq E\left(-\frac{\lambda^{2}}{2} n^{n+H_{1}-1} \sum_{j=0}^{n-1} \sum_{k=0}^{j-1} K\left(n^{\alpha} S_{j}^{H_{1}}\right)\right. \\
\left.\times K\left(n^{\alpha} S_{k}^{H_{1}}\right) f_{H_{2}}(j, k)\right) .
\end{gathered}
$$

We get the following result again which is now valid for $\mathrm{H}_{2} \in$ $(0,1)$ :

$$
E\left(\left|J_{n}^{(2)}\right|\right) \longrightarrow 0, \quad \text { as } n \longrightarrow \infty
$$

Concerning the term $J_{n}^{(1)}$ we note that

$$
E\left(\left|J_{n}^{(1)}\right|\right)=E\left(e^{-\left(\lambda_{n} / 2\right)\langle S\rangle_{n}}\right),
$$

and the result follows from Theorem 8 .

\section{Conflict of Interests}

The authors declare that there is no conflict of interests regarding the publication of this paper.

\section{Acknowledgments}

The authors want to thank the editor and anonymous referees whose valuable remarks and suggestions greatly improved the presentation of this paper. The project is sponsored by NSFC (no. 11226198 and no. 11171062), Innovation Program of Shanghai Municipal Education Commission (12ZZ063), and Priority Academic Program Development of Jiangsu Higher Education Institutions.

\section{References}

[1] L. P. Hansen, "Large sample properties of generalized method of moments estimators," Econometrica, vol. 50, pp. 1029-1054, 1982.

[2] D. W. K. Andrewa and C. J. McDermott, "Nonlinear econometric models with deterministically trending variables," Reiew of Economic Studies, vol. 62, pp. 343-360, 1995.

[3] J. M. Wooldridge, "Chapter 45 Estimation and inference for dependent processes," Handbook of Econometrics, vol. 4, pp. 2639-2738, 1994. 
[4] C. W. J. Granger, "Nonlinear relationships between extendedmemory variables," Econometrica, vol. 63, no. 2, pp. 265-280, 1995.

[5] H. A. Karlsen, T. Myklebust, and D. Tjøstheim, "Nonparametric estimation in a nonlinear cointegration type model," Annals of Statistics, vol. 35, no. 1, pp. 252-299, 2007.

[6] Q. Wang and P. C. B. Phillips, "Asymptotic theory for local time density estimation and nonparametric cointegrating regression," Econometric Theory, vol. 25, no. 3, pp. 710-738, 2009.

[7] S. Bourguin and C. A. Tudor, "Asymptotic theory for fractional regression models via malliavin calculus," Journal of Theoretical Probability, vol. 25, no. 2, pp. 536-564, 2012.

[8] L. Yan and G. Shen, "On the collision local time of sub-fractional Brownian motions," Statistics and Probability Letters, vol. 80, no. 5-6, pp. 296-308, 2010.

[9] L. Yan, G. Shen, and K. He, "Itô's formula for a sub-fractional Brownian motion," Communications on Stochastic Analysis, vol. 5, no. 1, pp. 135-159, 2011.

[10] T. Bojdecki, L. G. Gorostiza, and A. Talarczyk, "Fractional Brownian density process and its self-intersection local time of order k," Journal of Theoretical Probability, vol. 17, no. 3, pp. 717739, 2004.

[11] T. Bojdecki, L. G. Gorostiza, and A. Talarczyk, "Limit theorems for occupation time fluctuations of branching systems I: longrange dependence," Stochastic Processes and their Applications, vol. 116, no. 1, pp. 1-18, 2006.

[12] T. Bojdecki, L. G. Gorostiza, and A. Talarczyk, "Some extensions of fractional brownian motion and sub-fractional browian motion related to particle systems," Electronic Communications in Probability, vol. 12, pp. 161-172, 2007.

[13] J. Liu and L. Yan, "Remarks on asymptotic behavior of weighted quadratic variation of subfractional Brownian motion," Journal of the Korean Statistical Society, vol. 41, no. 2, pp. 177-187, 2012.

[14] J. Liu, L. Yan, Z. Peng, and D. Wang, "Remarks on confidence intervals for self-similarity parameter of a subfractional brownian motion," Abstract and Applied Analysis, vol. 2012, Article ID 804942, 14 pages, 2012.

[15] C. Tudor, "Inner product spaces of integrands associated to subfractional Brownian motion," Statistics and Probability Letters, vol. 78, no. 14, pp. 2201-2209, 2008.

[16] D. Nualart, Malliavin Calculus and Related Topics, Springer, New York, NY, USA, 2nd edition, 2006.

[17] C. Tudor, "Some properties of the sub-fractional Brownian motion," Stochastics, vol. 79, no. 5, pp. 431-448, 2007.

[18] M. Eddahbi, R. Lacayo, J. L. Solé, J. Vives, and C. A. Tudor, "Regularity of the local time for the $d$ fractional Brownian motion with N," Stochastic Analysis and Applications, vol. 23, no. 2, pp. 383-400, 2005. 


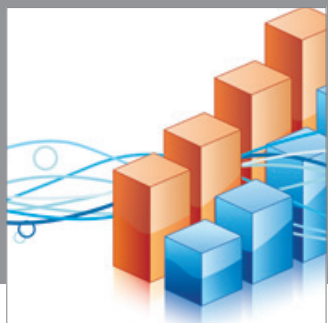

Advances in

Operations Research

mansans

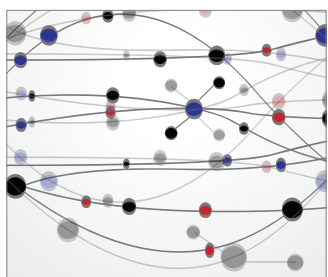

The Scientific World Journal
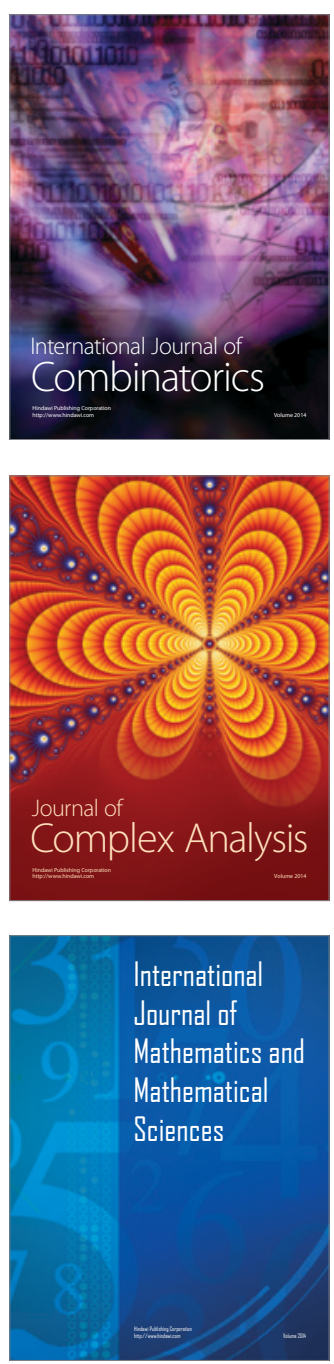
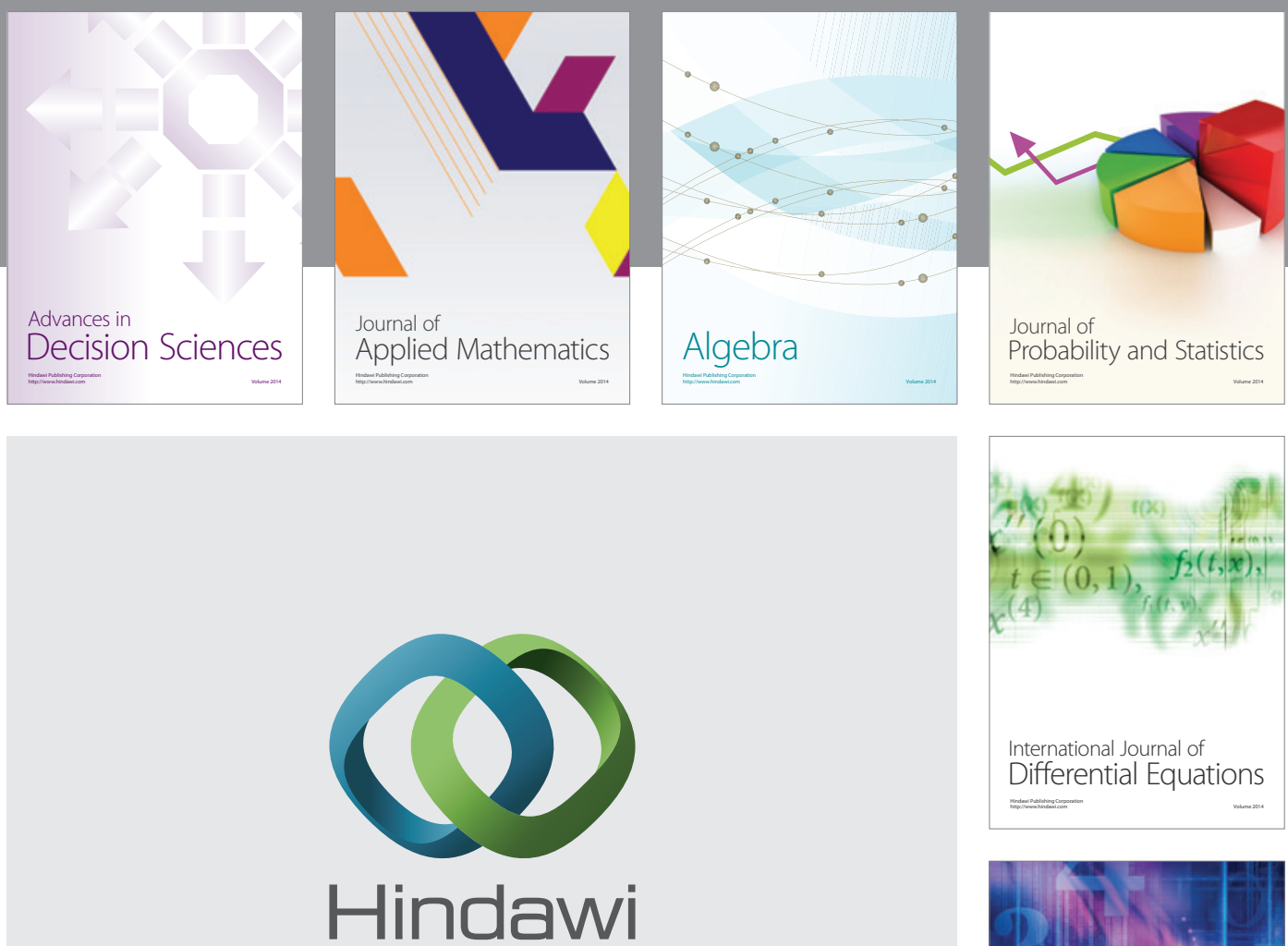

Submit your manuscripts at http://www.hindawi.com
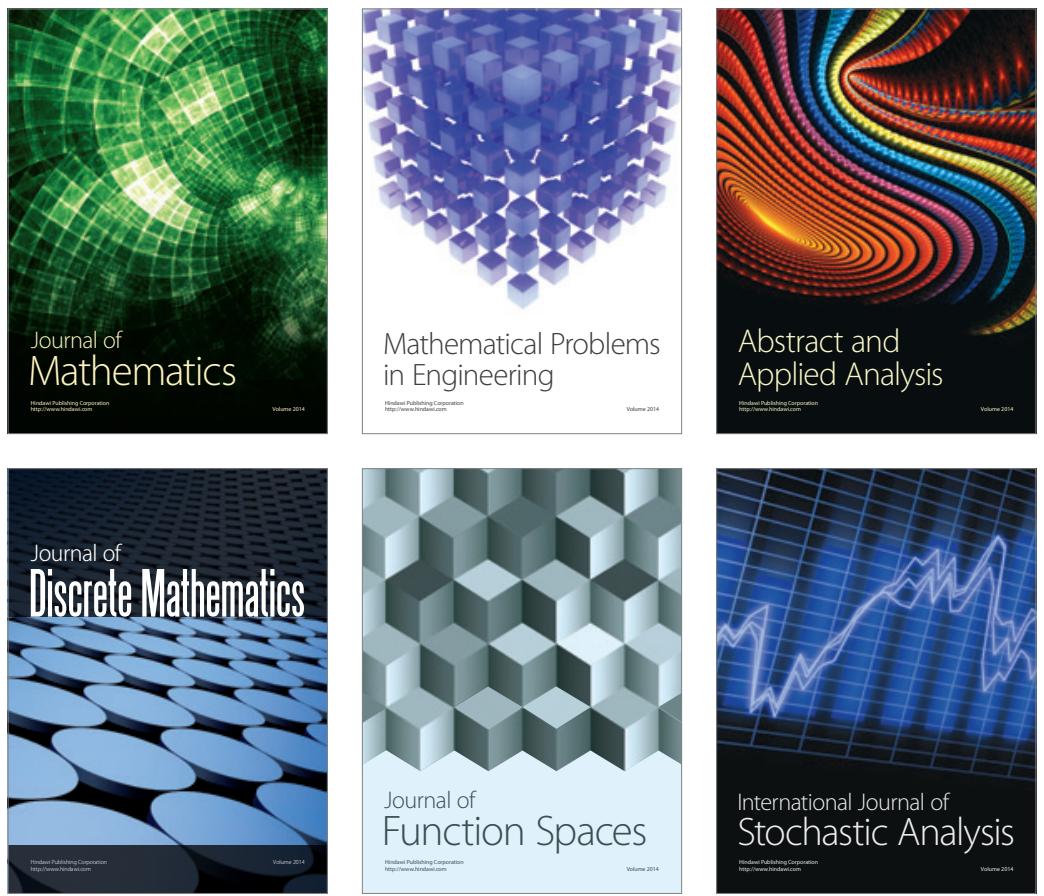

Journal of

Function Spaces

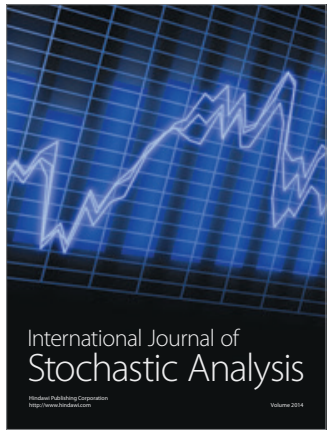

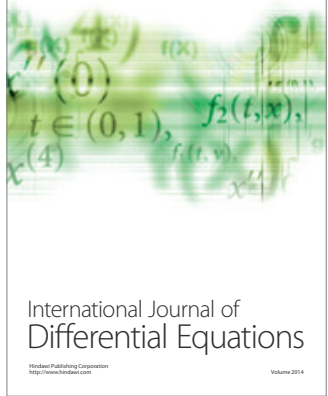
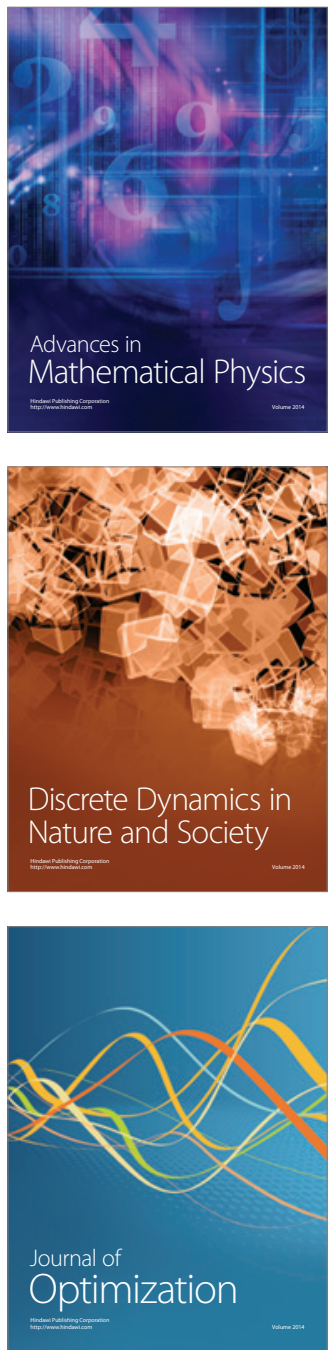